\title{
The burden and risk factors of ventriculostomy occlusion in a high-volume cerebrovascular practice: results of an ongoing prospective database
}

\author{
Kyle M. Fargen, MD, MPH, ${ }^{1}$ Brian L. Hoh, MD, ${ }^{1}$ Dan Neal, MS, ${ }^{1}$ Timothy O'Connor, MD, ${ }^{1}$ \\ Marie Rivera-Zengotita, MD, ${ }^{2}$ and Gregory J. A. Murad, MD' \\ Departments of ${ }^{1}$ Neurosurgery and ${ }^{2}$ Pathology and Laboratory Medicine, University of Florida, Gainesville, Florida
}

OBJECTIVE Ventriculostomy occlusion is a known complication after external ventricular drain (EVD) placement. There have been no prospective published series that primarily evaluate the incidence of and risk factors for EVD occlusion. These phenomena are investigated using a prospective database.

METHODS An ongoing prospective study of all patients undergoing frontal EVD placement in the Neurosurgery Intensive Care Unit at the University of Florida was accessed for the purposes of this analysis. Demographic, procedural, and radiographic data were recorded prospectively and retrospectively. The need for catheter irrigation or replacement was meticulously documented. Univariate and multivariate regression analyses were performed.

RESULTS Ninety-eight of 101 total enrolled patients had accessible data, amounting to 131 total catheters and 1076 total catheter days. Nineteen percent of patients required at least 1 replacement. Forty-one percent of catheters developed at least 1 temporary occlusion, with an average of 2.4 irrigations per patient. Intracranial hemorrhage occurred in $28 \%$ of patients after the first EVD placement ( $2 \%$ resulting in new neurological deficit) and in $62 \%$ of patients after 1 replacement. The cost of occlusion is estimated at $\$ 615$ per enrolled patient. Therapeutic anticoagulation and use of small EVD catheters were statistically significant predictors of permanent occlusion ( $p=0.01$ and 0.04 , respectively).

CONCLUSIONS EVD occlusion is frequent and imparts a significant burden in terms of patient morbidity, physician upkeep, and cost. This study suggests that developing strategies or devices to prevent EVD occlusion, such as the preferential use of larger diameter catheters, may be beneficial in reducing the burden associated with ventriculostomy malfunction.

http://thejns.org/doi/abs/10.3171/2015.5.JNS15299

KEY WORDS external ventricular drain; hydrocephalus; irrigation; occlusion; subarachnoid hemorrhage; ventriculostomy; vascular disorders

$\mathrm{V}$ ENTRICULOSTOMY occlusion is a common complication after external ventricular drain (EVD) placement. A review of published prospective and retrospective studies indicates that approximately $1 \%$ to $7 \%$ of EVDs require replacement secondary to occlusion. . $^{1-3,5,8,9,20}$ Furthermore, in a Nationwide Inpatient Sample database study of more than 101,000 hospitalizations in which ventriculostomy was performed, nearly $6 \%$ of patients required at least 1 additional ventriculostomy procedure. ${ }^{18}$ Studies have demonstrated nonpatent EVD in 19\% to $47 \%$ of patients; $3,4,15$ however, no study to date has prospectively evaluated EVD occlusion as a primary end point, re- ported on the number of catheter irrigations performed, or identified the risk factors for EVD occlusion.

The morbidity and costs associated with the occlusion of EVD catheters is not insignificant. Patients with nonfunctional catheters often require CT imaging to evaluate the catheter position, subjecting the patient to ionizing radiation. Replacement of EVD catheters may be costly and uncomfortable for patients. In addition, studies have documented an increased risk of ventriculostomy infection with replacement. ${ }^{4,12}$ Finally, catheter irrigation performed to restore the patency of occluded catheters may not be a benign maneuver (unpublished results). 
As part of an ongoing prospective database of EVD placement at our institution, we have monitored the incidence of temporary and permanent catheter occlusion, need for replacement, and frequency of catheter irrigation. The purpose of this study is to describe the burden of EVD occlusion in a prospective manner and identify the risk factors associated with ventriculostomy occlusion.

\section{Methods \\ Study Database}

An ongoing, prospective, institutional review boardapproved study of all patients undergoing EVD placement in the neurosurgical intensive care unit (ICU) at our institution was accessed for the purposes of this analysis. The ongoing prospective database was initiated in August 2013 and has, to date, enrolled 101 patients. This analysis represents data collected for 1 year of enrollment.

All adult patients receiving bedside EVD placement in the neurosurgical ICU by a neurosurgical resident physician were candidates. Patients or next of kin provided consent for inclusion on a prospective basis. Patients transferred from other institutions in which an EVD had already been placed, or those patients with an EVD placed in the operating room, were excluded. Furthermore, EVDs placed in the trauma ICU were excluded due to the inability to accurately obtain occlusion data due to different nursing protocols. Therefore, the majority of traumatic brain injury patients who received EVD were excluded. Overall, enrolled patients accounted for approximately $50 \%$ of all patients receiving any EVD at our institution and about two-thirds of all patients undergoing bedside EVD placement.

\section{Procedural Technique}

All EVDs were placed by resident physicians in the neurosurgical ICU using a previously published protocol. ${ }^{17}$ This protocol includes using a bur hole entry point $1 \mathrm{~cm}$ anterior to the coronal suture in the midpupillary line, full preparation and craniotomy drape, preprocedural antibiotic administration, and tunneling the catheter to an exit site at least $5 \mathrm{~cm}$ from the incision. Two Codman (Codman \& Shurtleff Inc.) catheters were available for use (Clear Bactiseal "large" catheter: 3.4-mm outer diameter and 1.9$\mathrm{mm}$ inner diameter; and Orange Bactiseal "small" catheter: $3-\mathrm{mm}$ outer diameter and $1.5-\mathrm{mm}$ inner diameter). The decision regarding which catheter to use was made at the time of the procedure based on resident preference and availability.

\section{Irrigation Procedure}

No formal published protocols exist for catheter irrigation. There is no standard policy for EVD irrigation at our institution, and the specifics regarding the procedure are not recorded in nursing or physician notes. In general, resident physicians are instructed to first attempt distal irrigation of the drainage chamber using sterile techniques (rarely effective), followed by gentle aspiration of the proximal system and catheter if distal flushing is not effective. If these do not restore patency, a small volume of sterile saline, usually $3 \mathrm{ml}$ or less, is flushed proximally into the catheter. However, the volume infused, the injection force, the aspiration force, and the number of attempts are left to individual physician discretion as they are not supervised.

\section{Data Collection}

Data were collected both prospectively and retrospectively from a prospective database. Collected data included a number of factors, such as demographics and underlying diagnosis; procedural data including the postgraduate year of training of the proceduralist; number of passes, side of placement, and type of catheter used; radiographic data including ventricular size (based on the Evans ratio: maximal bifrontal ventricular distance divided by maximal intracranial biparietal distance), preprocedural midline shift, presence of intraventricular hemorrhage, catheter location, and presence of catheter-related hemorrhage; and clinical data including symptomatic hemorrhage, number and type of catheter irrigations, catheter occlusion and replacement, number of catheter days, catheter endpoint, cerebrospinal fluid analysis, and incidence of catheter-related infection. Preoperative therapeutic anticoagulation was defined by the presence of 1 or more of the following active medications, either based on laboratory studies or the use of reversal agents prior to the procedure: warfarin, clopidogrel, prasugrel, therapeutic Lovenox, or heparin infusion. The catheter position was characterized as 1) catheter tip within $5 \mathrm{~mm}$ of the ipsilateral foramen of Monro (FoM); 2) catheter in the ipsilateral lateral ventricle but $\geq 6 \mathrm{~mm}$ from FoM; 3) tip in the third ventricle; 4) tip in the contralateral lateral ventricle; or 5) catheter not within ventricle. The "ideal" catheter position was defined as the catheter within $5 \mathrm{~mm}$ of ipsilateral FoM, "acceptable" position as the catheter in the ipsilateral lateral ventricle or third ventricle, and "suboptimal" position as the catheter in the contralateral lateral ventricle or outside of the ventricular system. Preprocedural midline shift was further dichotomized as $>5 \mathrm{~mm}$ and $\leq 5 \mathrm{~mm}$. Postprocedural intracranial hemorrhage was characterized by size and location.

Patients met the study endpoint when the EVD catheter was removed or replaced by a ventricular shunt. Temporary occlusion was defined as a nonpatent EVD requiring neurosurgeon irrigation that successfully restored patency. Permanent occlusion was defined as a nonpatent EVD where patency could not be successfully restored through irrigation. Temporary occlusions were identified through reviewing both nurse and physician documentation. The number of CT scans clearly identified in the physician notes as ordered solely for the purposes of checking catheter position due to nonfunctioning EVD were recorded for each patient. CT scans obtained for mental status changes, preoperative planning for ventriculoperitoneal shunting, or other reasons were excluded from the cost analysis.

\section{Cost Analysis}

The hospital charges for all supplies used during EVD replacement, including catheters, preparation and drapes, cranial access kits, preoperative antibiotics, procedural supplies, and drainage systems, were totaled to obtain an estimate of the total cost of performing a replacement (approximately $\$ 1035)$. The obtained brain CT scans were 
TABLE 1. Demographic and procedural data

\begin{tabular}{|c|c|}
\hline Factor & Value* $^{*}$ \\
\hline Age, yrs [mean, range (SEM)] & $59.5,19-91(14.5)$ \\
\hline \multicolumn{2}{|l|}{ Sex } \\
\hline Male & $43(43.4 \%)$ \\
\hline Female & $56(56.6 \%)$ \\
\hline \multicolumn{2}{|l|}{ Diagnosis } \\
\hline Subarachnoid hemorrhage & $64(64.6 \%)$ \\
\hline Meningitis or ventriculitis & $5(5.1 \%)$ \\
\hline All others & $30(30.3 \%)$ \\
\hline Presence of therapeutic anticoagulation & $15(15.2 \%)$ \\
\hline \multicolumn{2}{|l|}{ Preprocedure ventricular size, Evan ratio } \\
\hline Mean (SEM) & $0.31(0.053)$ \\
\hline Range & $0.07-0.46$ \\
\hline \multicolumn{2}{|l|}{ Preprocedural intraventricular hemorrhage } \\
\hline None & $38(38.4 \%)$ \\
\hline Yes & $61(61.6 \%)$ \\
\hline Blood in all 4 ventricles & $12(19.7 \%)$ \\
\hline Blood in ipsilateral LV & $5(8.2 \%)$ \\
\hline Blood in contralateral LV, 3rd or 4th & $22(36.1 \%)$ \\
\hline Blood settling in the atrium of LV & $22(36.1 \%)$ \\
\hline Presence of endotracheal tube & $47(47.5)$ \\
\hline \multicolumn{2}{|l|}{ Midline shift, mm } \\
\hline Mean (SEM) & $1.2(2.8)$ \\
\hline Range & $0-12$ \\
\hline Midline shift $\geq 5 \mathrm{~mm}$ & $13(13.1 \%)$ \\
\hline \multicolumn{2}{|l|}{ CSF studies (obtained after EVD placement) } \\
\hline No & $39(39.4 \%)$ \\
\hline Yes & $60(60.6 \%)$ \\
\hline \multicolumn{2}{|l|}{ RBC, no. } \\
\hline Mean (SEM) & 467,000 (2.78 million) \\
\hline Range & 2-21 million \\
\hline \multicolumn{2}{|l|}{ WBC, no. } \\
\hline Mean (SEM) & $898(2350)$ \\
\hline Range & $0-16,000$ \\
\hline \multicolumn{2}{|l|}{ Protein, mg/dl } \\
\hline Mean (SEM) & $101(114)$ \\
\hline Range & $7-594$ \\
\hline \multicolumn{2}{|l|}{ Glucose, mg/dl } \\
\hline Mean (SEM) & $79.1(21.7)$ \\
\hline Range & $2-125$ \\
\hline \multicolumn{2}{|l|}{$\%$ PMN } \\
\hline Mean (SEM) & $66.4 \%(24.9 \%)$ \\
\hline Range & $2-100 \%$ \\
\hline \multicolumn{2}{|l|}{ RBC/WBC ratio } \\
\hline Mean (SEM) & $1270(6450)$ \\
\hline Range & $0-49,100$ \\
\hline
\end{tabular}

(continued)
TABLE 1. Demographic and procedural data (continued)

\begin{tabular}{lc}
\hline \multicolumn{1}{c}{ Factor } & Value $^{*}$ \\
\hline Procedural data & \\
\hline Side & $77(77.8 \%)$ \\
\hline Right & $22(22.2 \%)$ \\
\hline Left & \\
\hline Catheter used & $53(53.5 \%)$ \\
\hline Small & $46(46.5 \%)$ \\
\hline Large & $1.3(0.82)$ \\
\hline No. of passes & $1-5$ \\
\hline Mean (SEM) & \\
\hline Range & $52(52.3 \%)$ \\
\hline PGY of the resident & $43(43.4 \%)$ \\
\hline $1-2$ & $4(4.0 \%)$ \\
\hline $3-4$ &
\end{tabular}

$\mathrm{LV}=$ lateral ventricle; $\mathrm{PGY}=$ postgraduate year; $\mathrm{PMN}=$ polymorphonuclear leukocyte; RBC = red blood cells; $\mathrm{WBC}=$ white blood cells.

* Values are presented as the number of patients (\%) unless specified otherwise.

estimated at approximately $\$ 900$ per study, including the fee for radiology review.

\section{Statistical Analysis}

The R statistical software package (V.3.0.2) was used for analysis. Univariable and multivariable logistic regression analyses were performed to determine significant predictors. Kaplan-Meier curves were created to show differences in catheter survival.

\section{Results}

\section{Demographic and Preprocedural Data}

A total of 101 patients were enrolled in the study. Two patients were excluded due to improper informed consent. One patient was concurrently enrolled in a trial involving the routine intrathecal administration of medication, and therefore this patient was excluded from the occlusion analysis due to potential confounding from routine irrigation for nonocclusion. Table 1 contains detailed demographic, preprocedural, and procedural data for the included patients. The vast majority of patients received the EVD after subarachnoid hemorrhage. Five patients had meningitis or ventriculitis prior to EVD placement. All 15 patients with therapeutic anticoagulation were administered standard-of-care reversal agents prior to EVD placement.

\section{Postprocedural Data}

Overall, the study included 1076 catheter days (mean 10.9 days per patient; range 1-43 days; SEM 7.2 days). Of the 98 patients, 41 (42\%) developed at least 1 temporary occlusion requiring irrigation and 19 developed at least 1 permanent occlusion (19\%). Overall, 131 catheters were followed in the 98 patient sample. A total of 235 irriga- 
tions were performed among the 98 patients (mean 2.4 flushes per enrolled patient; range 0-45 flushes; SEM 6.1 flushes) or 1.8 flushes per catheter. A total of 33 replacements were performed among these 19 patients (mean 0.34 replacements per enrolled patient; range $0-7$ replacements; SEM 1.0 replacement), amounting to 0.25 replacements per catheter.

Table 2 contains the detailed postprocedural radiographic and clinical data. Postprocedural imaging was obtained in 90 patients $(92 \%)$. A total of 29 CT scans were obtained during the study period solely for the purpose of checking catheter position with occlusion (mean 0.30 scans per patient; range 0-6 scans; SEM 0.9 scans). Ideal catheter position was seen in $33.3 \%$ of patients, and an acceptable position was seen in $84.5 \%$ of patients. New intracranial hemorrhage was discovered in 25 patients after virgin EVD placement (27.8\%); the majority of new hemorrhages $(68 \%)$ were $\leq 5 \mathrm{~mm}$ parenchymal hemorrhages around the catheter. There were 5 symptomatic hemorrhages on virgin EVD placement (5.1\%): 3 hemorrhages resulting in near-immediate catheter occlusion necessitating replacement, and 2 large parenchymal hemorrhages resulting in contralateral hemiparesis. Excluding those resulting in replacement, there was a $2 \%$ risk of symptomatic hemorrhage. No new hemorrhages requiring craniotomy for hematoma evacuation occurred during the study period. Of the 19 patients who underwent at least 1 replacement, imaging was available after the first replacement in 13 patients (68\%); 8 of these 13 patients $(62 \%)$ had evidence of new hemorrhage on repeat imaging. There was no relationship between catheter size and new hemorrhage $(\mathrm{p}=1.0)$. There were no instances of new meningitis or ventriculitis.

\section{Temporary Occlusion Analysis}

Table 3 demonstrates the results of the univariable analysis of potential predictors of 1 or more temporary occlusions. The catheter used $(\mathrm{p}=0.10)$, presence of therapeutic anticoagulation $(\mathrm{p}=0.05)$, dichotomized preprocedural midline shift ( $\mathrm{p}=0.07)$, and nonideal catheter position ( $\mathrm{p}$ $=0.03$ ) were selected for multivariable analysis. Sex was excluded from the model due to a high correlation with anticoagulation $(p=0.002)$. Table 4 demonstrates the results of the multivariable analysis that used these factors. All 4 factors (small catheter, therapeutic anticoagulation, midline shift greater than $5 \mathrm{~mm}$, and nonideal position) demonstrated a strong trend toward being predictive of temporary occlusion, although none were statistically significant.

\section{Permanent Occlusion Analysis}

Table 3 demonstrates the results of the univariable analysis of potential predictors of 1 or more permanent occlusions. Catheter used $(p=0.07)$, presence of therapeutic anticoagulation $(\mathrm{p}=0.01)$, and nonideal catheter position $(\mathrm{p}=0.05)$ were selected for the multivariable analysis. Intracranial hemorrhage (ICH) was excluded from this analysis due to a strong correlation between therapeutic anticoagulation and ICH (62\% of anticoagulated patients had ICH compared with only $22 \%$ of those not anticoagulated; $p=0.006$ ). Table 4 demonstrates the results of the
TABLE 2. Postprocedural data, including catheter position, intracranial hemorrhage, and occlusion data

\begin{tabular}{|c|c|}
\hline Factor & Value $^{*}$ \\
\hline \multicolumn{2}{|l|}{ Postprocedural imaging performed } \\
\hline None & $9(9.1 \%)$ \\
\hline CT & $82(82.8 \%)$ \\
\hline MRI & $8(8.1 \%)$ \\
\hline \multicolumn{2}{|l|}{ Catheter position } \\
\hline Ipsilateral LV $\leq 5 \mathrm{~mm}$ from FoM & $30(33.3 \%)$ \\
\hline Ipsilateral LV >5 mm from FoM & $37(41.1 \%)$ \\
\hline 3rd ventricle & $9(10.0 \%)$ \\
\hline Contralateral LV & $11(12.2 \%)$ \\
\hline Extraventricular & $3(3.3 \%)$ \\
\hline \multicolumn{2}{|c|}{$\begin{array}{l}\text { New intracranial hemorrhage on 1st postproce- } \\
\text { dure imaging }\end{array}$} \\
\hline None & $65(72.2 \%)$ \\
\hline$\leq 5 \mathrm{~mm}$ parenchymal & $17(18.9 \%)$ \\
\hline$>5 \mathrm{~mm}$ parenchymal & $4(4.4 \%)$ \\
\hline Subdural hematoma & $0(0)$ \\
\hline Intraventricular hemorrhage & $2(2.2 \%)$ \\
\hline Multiple hemorrhage types & $2(2.2 \%)$ \\
\hline \multicolumn{2}{|l|}{ Endpoint of final EVD catheter } \\
\hline Discontinued without shunt placement & $50(50.5 \%)$ \\
\hline Conversion to shunt & $27(27.3 \%)$ \\
\hline Discontinued accidentally & $2(2.0 \%)$ \\
\hline Patient deceased & $19(19.2 \%)$ \\
\hline Transferred to other hospital in place & $1(1.0 \%)$ \\
\hline \multicolumn{2}{|l|}{ Total catheter days } \\
\hline Mean (SEM) & $10.9(7.2)$ \\
\hline Range & $1-43$ \\
\hline \multicolumn{2}{|l|}{ Occlusion data per patient enrolled } \\
\hline \multicolumn{2}{|l|}{ No. of irrigations } \\
\hline Mean (SEM) & $2.4(6.1)$ \\
\hline Range & $0-45$ \\
\hline \multicolumn{2}{|l|}{ No. of replacements } \\
\hline Mean (SEM) & $0.34(1.0)$ \\
\hline Range & $0-7$ \\
\hline \multicolumn{2}{|l|}{ No. of CT scans to check placement } \\
\hline Mean (SEM) & $0.30(0.86)$ \\
\hline Range & $0-6$ \\
\hline
\end{tabular}

* Values are presented as the number of patients (\%) unless specified otherwise.

multivariable analysis using these 3 factors. Patients who received a small catheter had a 3.4 times greater odds of developing occlusion compared with patients receiving large catheters. Similarly, patients who were therapeutically anticoagulated had a 6.7 times greater odds of developing occlusion than patients who were not anticoagulated. Patients with catheters more than $5 \mathrm{~mm}$ from the ipsilateral FoM had a 4.2 times greater odds of occlusion compared with patients with catheters within this radius, although this was only marginally significant $(\mathrm{p}=0.054)$. 
TABLE 3. Results of the univariable analyses for both temporary and permanent occlusion

\begin{tabular}{lcc}
\hline \multicolumn{1}{c}{ Factor } & $\begin{array}{c}\text { Temporary } \\
\text { Occlusion } \\
\text { p Value }\end{array}$ & $\begin{array}{c}\text { Permanent } \\
\text { Occlusion } \\
\text { p Value }\end{array}$ \\
\hline Age & 0.48 & 0.99 \\
\hline Sex & 0.06 & 0.61 \\
\hline Diagnosis type & 0.36 & 0.43 \\
\hline IVH (dichotomous) & 0.80 & 0.44 \\
\hline IVH type (categorical) & 0.90 & 0.79 \\
\hline Catheter used & 0.10 & 0.07 \\
\hline Presence of ETT & 0.41 & 0.62 \\
\hline Side of procedure & 0.81 & 0.76 \\
\hline Evans ratio & 0.12 & 0.22 \\
\hline Midline shift (linear) & 0.08 & 0.65 \\
\hline Midline shift (dichotomous) & 0.07 & 1.0 \\
\hline No. of passes & 0.29 & 0.75 \\
\hline Therapeutic anticoagulation & 0.05 & 0.01 \\
\hline Nonideal catheter position & 0.03 & 0.05 \\
\hline Unacceptable catheter position & 0.39 & 0.15 \\
\hline Postprocedural ICH & 0.34 & 0.02 \\
\hline CSF studies* & $0.09-0.52$ & $0.11-0.84$ \\
\hline
\end{tabular}

ETT = endotracheal tube.

* Includes CSF red blood cell, white blood cell, protein, glucose, percentage polymorphonuclear leukocyte, and RBC/WBC ratio.

\section{Cost Analysis}

Based on an average cost of $\$ 1035$ for replacement, the total cost of replacement among the 98-patient cohort was $\$ 34,155$. Based on an average of $\$ 900$ for each CT scan obtained solely for the purposes of checking the position of an occluded catheter, the total cost of CT for occlusion was $\$ 26,100$. Overall, the total cost of occlusion was $\$ 60,255$, or approximately $\$ 615$ per enrolled patient.

\section{Catheter Analysis}

Postprocedural ICH was seen in $28 \%$ of patients after the implantation of either a small or large catheter. No statistical difference in the hemorrhage rate was identified ( $\mathrm{p}$ $=1.0$ ). There were no identified differences in patient factors between those who received small catheters or large catheters (Table 5).

Thirteen of 51 small catheters became permanently occluded (25.5\%). The estimated mean survival time for small catheters is 20.5 days (95\% CI 17.3-23.7 days), with the mean restricted to 27.5 days (based on the maximum follow-up). Five permanent occlusions occurred in 46 large catheters $(10.9 \%)$. The estimated mean survival time for large catheters is 24.7 days (95\% CI $22.4-27.0$ days), with the mean restricted to 27.5 days. Log-rank analysis demonstrates that catheter survival is longer for large catheters compared with small catheters $(p=0.05)$. Figure 1 demonstrates the Kaplan-Meier survival curve comparing catheter size. All 19 initial catheters that progressed to permanent occlusion did so within the first 10 days of implantation.
TABLE 4. Results of the multivariable analysis of temporary and permanent occlusion

\begin{tabular}{lll}
\hline \multicolumn{1}{c}{ Factor } & Odds Ratio $(95 \% \mathrm{Cl})$ & $\mathrm{p}$ Value \\
\hline Temporary occlusion & & \\
\hline Small catheter & $2.2(0.87-5.5)$ & 0.10 \\
\hline Therapeutic anticoagulation & $3.2(0.89-13.4)$ & 0.09 \\
\hline Midline shift $>5 \mathrm{~mm}$ & $3.3(0.91-13.6)$ & 0.06 \\
\hline Nonideal position & $2.6(0.97-7.5)$ & 0.07 \\
\hline Permanent occlusion & & \\
\hline Small catheter & $3.4(1.03-13.2)$ & 0.04 \\
\hline Therapeutic anticoagulation & $6.7(1.73-28.2)$ & 0.01 \\
\hline Nonideal position & $4.2(0.98-29.2)$ & 0.05 \\
\hline
\end{tabular}

\section{Discussion}

This prospective database encompassing nearly 1100 catheter days in a high-volume cerebrovascular practice represents the most rigorous study to date to evaluate the burden and risk factors for ventriculostomy occlusion. Overall, the burden of EVD occlusion in terms of physician maintenance, patient morbidity, and cost is higher than expected. Nearly 1 in 5 patients enrolled in this study required at least 1 replacement; 1 in every 4 catheters became permanently occluded. Over $40 \%$ of catheters developed at least 1 temporary occlusion, with an average of 2.4 irrigations per patient or 1.8 irrigations per catheter.

Additionally, although more passes during placement has been associated with a greater risk of hemorrhage, ${ }^{7}$ this is the first study to prospectively report on the risk of hemorrhage associated with the replacement of a second catheter. New intracranial hemorrhage was seen in $28 \%$ of patients after virgin catheter placement and in $62 \%$ after 1 replacement, although a very small number resulted in neurological symptoms (2\%). Approximately 1 in every 4 patients underwent an additional CT scan of the brain, and received the radiation dose associated with that study, solely to evaluate position after catheter occlusion. The cost of occlusion in terms of supplies and CT imaging was more than $\$ 60,000$ for the 98 enrolled patients, or more than $\$ 600$ per patient. Overall, this study clearly documents that bedside EVD occlusion imparts significant burden onto both patients and the neurosurgeons managing these devices. Furthermore, this study suggests that developing strategies or devices to prevent EVD occlusion may be beneficial in reducing the burden associated with ventriculostomy malfunction.

This study is also the first study to rigorously evaluate the risk factors for EVD occlusion. While there were no statistically significant predictors of temporary occlusion, 4 factors (small catheter, therapeutic anticoagulation, midline shift greater than $5 \mathrm{~mm}$, and nonideal position) all showed trends $(\mathrm{p}=0.056-0.10)$ toward increasing temporary occlusion risk. Most importantly, 2 of these factors were statistically significant predictors of permanent occlusion (small catheter and therapeutic anticoagulation), imparting 3 times and 6 times greater odds of occlusion, respectively. Nonideal catheter position was once again marginally significant in this analysis. The reasons for the 
TABLE 5. Comparison of patient and procedural factors by catheter used

\begin{tabular}{|c|c|c|c|}
\hline Factor & $\begin{array}{c}\text { Small } \\
\text { Catheter* }^{*}\end{array}$ & $\begin{array}{l}\text { Large } \\
\text { Catheter* }^{*}\end{array}$ & $\begin{array}{c}p \\
\text { Value }\end{array}$ \\
\hline \multicolumn{4}{|l|}{ Demographic factors } \\
\hline Mean age in yrs (SD) & $58.4(15.7)$ & $60.8(12.9)$ & 0.69 \\
\hline \multicolumn{4}{|l|}{ Sex } \\
\hline Male & $20(37.7 \%)$ & $23(50.0 \%)$ & \multirow[t]{2}{*}{0.23} \\
\hline Female & $33(62.3 \%)$ & $23(50.0 \%)$ & \\
\hline \multicolumn{4}{|l|}{ Diagnosis type } \\
\hline $\begin{array}{l}\text { Subarachnoid hemor- } \\
\text { rhage }\end{array}$ & $34(64.2 \%)$ & $30(65.2 \%)$ & \multirow[t]{2}{*}{1.0} \\
\hline All others & $19(35.8 \%)$ & $16(34.8 \%)$ & \\
\hline \multicolumn{4}{|l|}{ IVH } \\
\hline No & $23(43.4 \%)$ & $15(32.6 \%)$ & \multirow[t]{2}{*}{0.31} \\
\hline Yes & $30(56.6 \%)$ & $31(67.4 \%)$ & \\
\hline \multicolumn{4}{|l|}{ Procedural factors } \\
\hline PGY (categorical) & & & 0.78 \\
\hline \multicolumn{4}{|l|}{$\begin{array}{l}\text { Presence of endotracheal } \\
\text { tube }\end{array}$} \\
\hline No & $29(54.7 \%)$ & $23(50.0 \%)$ & \multirow[t]{2}{*}{0.69} \\
\hline Yes & $24(45.3 \%)$ & $23(50.0 \%)$ & \\
\hline \multicolumn{4}{|l|}{ Side } \\
\hline Right & $39(73.6 \%)$ & $38(82.6 \%)$ & \multirow[t]{2}{*}{0.34} \\
\hline Left & $14(26.4 \%)$ & $8(17.4 \%)$ & \\
\hline Mean Evans ratio & $0.31(0.05)$ & $0.31(0.06)$ & 0.46 \\
\hline \multicolumn{4}{|l|}{ Midline shift } \\
\hline$<1 \mathrm{~mm}$ & $42(79.2 \%)$ & $39(84.8 \%)$ & \multirow[t]{2}{*}{0.60} \\
\hline$\geq 1 \mathrm{~mm}$ & $11(20.8 \%)$ & $7(15.2 \%)$ & \\
\hline \multicolumn{4}{|l|}{ No. of passes } \\
\hline 1 & $43(81.1 \%)$ & $38(82.6 \%)$ & \multirow[t]{2}{*}{1.0} \\
\hline$\geq 1$ & $10(18.9 \%)$ & $8(17.4 \%)$ & \\
\hline \multicolumn{4}{|l|}{ Any anticoagulation } \\
\hline No & $32(60.4 \%)$ & $36(78.3 \%)$ & \multirow[t]{2}{*}{0.08} \\
\hline Yes & $21(39.6 \%)$ & $10(21.7 \%)$ & \\
\hline \multicolumn{4}{|l|}{ Therapeutic anticoagulation } \\
\hline No & $44(83.0 \%)$ & $40(87.0 \%)$ & \multirow[t]{2}{*}{0.78} \\
\hline Yes & $9(17.0 \%)$ & $6(13.0 \%)$ & \\
\hline \multicolumn{4}{|l|}{ Ideal catheter position } \\
\hline Ideal & $15(31.9 \%)$ & $15(34.9 \%)$ & \multirow[t]{2}{*}{0.83} \\
\hline Not ideal & $32(68.1 \%)$ & $28(65.1 \%)$ & \\
\hline \multicolumn{4}{|l|}{ New ICH } \\
\hline No & $34(72.3 \%)$ & $31(72.1 \%)$ & \multirow[t]{2}{*}{1.0} \\
\hline Yes & $13(27.7 \%)$ & $12(27.9 \%)$ & \\
\hline
\end{tabular}

* Values are presented as the number of patients (\%) unless noted otherwise.

increased risks associated with these 4 factors have not been previously defined in the literature, but potential etiologies can be surmised. Anticoagulation has a strong association with intracranial hemorrhage, which could put the patient at higher risk for developing obstruction secondary to blood clot. It is important to note, however,

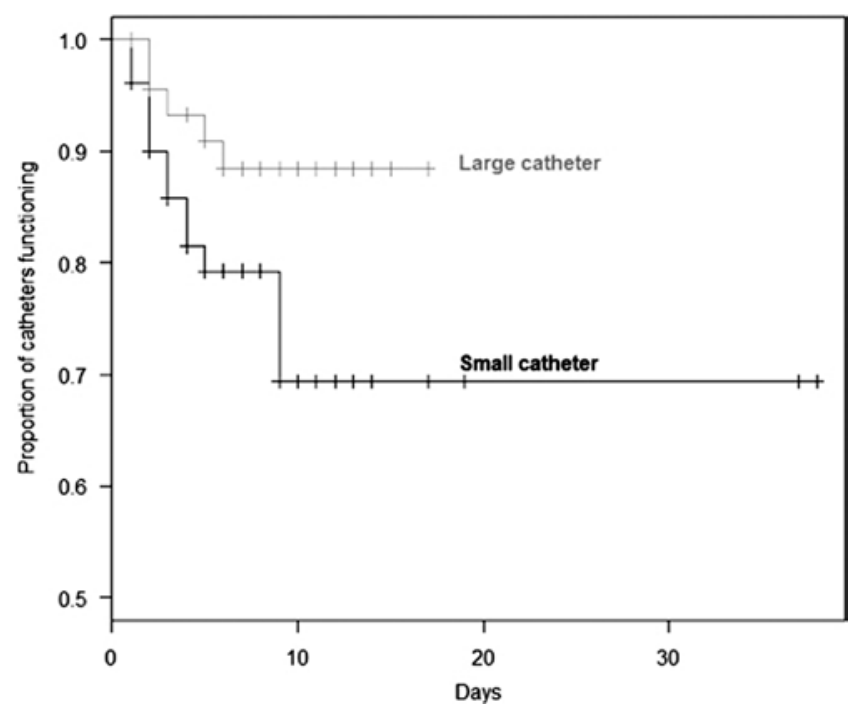

FIG. 1. Kaplan-Meier curve comparing catheter size on days to permanent occlusion.

that all 15 patients who had therapeutic anticoagulation on admission underwent appropriate reversal with standardof-care therapies, indicating that even with proper reversal an effect of these agents lingers. The small catheters, with only $79 \%$ of the internal diameter of the larger catheters, may have greater resistance to flow and more frequent obstructions as suggested by Poiseuille's law, where the radius has a tremendous effect on the pressure of a fluid column. Interestingly, studies have also suggested that larger catheters may result in smaller hemorrhage volumes compared with smaller catheters, possibly through a tamponade effect. ${ }^{14,19}$ Midline shift is a marker of unilateral pressure exerted on an unevenly distributed ventricular system in the setting of elevated intracranial pressure, which could result in ipsilateral ventricular decompression after EVD placement and obstruction of the catheter due to collapsed ventricular ependyma. In addition, this study validates the classic teaching of the FoM as the preferred target for EVD placement. Finally, no relationship was identified between the presence of preprocedural intraventricular hemorrhage (IVH) and eventual occlusion. While the role of IVH on occlusion may be limited by the small sample size of the study, this analysis does suggest that catheter occlusion remains a concern even in patients without IVH or hemorrhagic conditions.

While this study is the first prospective study designed to monitor the primary outcome of EVD occlusion, the results are, for the most part, consistent with published literature from retrospective series. The incidence of occlusion documented in this study falls within the range of previous studies that suggested nonpatent EVD in $19 \%$ to $47 \%$ of patients; $3,4,15$ however, the need for replacement is much higher than in previous retrospective studies, which have suggested a rate of $1 \%$ to $7 \% .^{1-3,5,8,9,20}$ The reasons for higher permanent occlusion documented in the present study are likely multifactorial, but could be related to more rigorous prospective monitoring and reporting of occlusion in this study in comparison with retrospective studies, which 
likely failed to identify a subset of occlusions due to inadequate documentation, and/or a high-volume academic neurosurgical patient population with a large proportion of subarachnoid hemorrhage and otherwise complex patients, which may be a higher risk patient population for occlusion. This effect is unlikely to be secondary to physician practices, as the incidence of acceptable catheter position is consistent with previous retrospective studies reporting $77 \%$ to $87 \%$ "acceptable" catheter position rates (although the definitions are somewhat different). ${ }^{7-9,16}$ Recent studies have indicated a $20 \%$ to $45 \%$ risk of hemorrhage with placement: $6,8,10,19$ the $28 \%$ hemorrhage incidence after virgin catheter placement in this study falls within this range. In this study, hemorrhage was determined based on the first postprocedural CT imaging. Given that some punctate hemorrhages have been observed only after EVD removal, ${ }^{6}$ it is therefore possible that some radiographic hemorrhages observed with replacement EVDs are caused by the removal of the first catheter rather than the placement of the second. Finally, the infection risk of 0 during this study is consistent with earlier findings from our ICU, further reinforcing the significance of infection control protocols with EVD placement. ${ }^{17}$

Because the burden and risk factors of EVD occlusion have not been rigorously reported to date, strategies for improving EVD occlusion are lacking in the published literature. Olson et al., in a randomized study comparing continuous versus intermittent drainage, showed a significantly lower occlusion rate with intermittent drainage than continuous drainage..$^{15}$ However, occlusion was poorly defined in this study and was not the primary outcome of this trial. The present study suggests that strategies for improving procedures that increase the incidence of ideal catheter position, as well as the preferential selection of larger diameter catheters, may potentially reduce the incidence of occlusion and need for replacement. Published studies have suggested that the use of bedside neuronavigation is feasible and may improve procedural accuracy.11,13 Expanded prospective studies will need to be performed before the benefits of these strategies can be confirmed.

\section{Limitations}

The incidence of temporary occlusion was dependent on nursing and physician documentation, so it is likely that the number of irrigations captured actually represents an underestimation of events. Conversely, the number of temporary occlusions may have been overestimated on some occasions, as multiple attempted irrigations may have been performed for the same permanent occlusion, and some of these events may have erroneously been recorded as temporary occlusions. We did not define a standard irrigation protocol and allowed the procedure to be based on the treating physician's discretion. In addition, this study only evaluates those EVDs placed at our institution in the neurosurgical ICU, and therefore these results may not apply to those placed in the operating room or through occipital trajectories. Furthermore, few traumatic brain injury patients were included as the majority of these patients were treated outside of the neurosurgical ICU. Permanent occlusion was infrequent (19 patients), thereby limiting the power of the multivariable analysis. Only $60 \%$ of patients had cerebrospinal fluid studies ordered, and therefore the study was underpowered to evaluate the effect of fluid contents on occlusion. Overall, however, the patient population followed in this study is likely to be very similar to patient populations at other high-volume academic institutions, and therefore the results presented are likely to be generalizable to other similar centers. Finally, catheters were chosen based on operator preference and not through randomization, so the effect of catheter size on occlusion may be influenced by selection bias.

\section{Conclusions}

EVD occlusion imparts a significant burden in terms of patient morbidity, physician upkeep, and cost. Nearly 1 in 5 patients enrolled in this study required at least 1 replacement; 1 in every 4 catheters became permanently occluded. Over $40 \%$ of catheters developed at least 1 temporary occlusion, with an average of 2.4 irrigations per patient or 1.8 irrigations per catheter. Furthermore, intracranial hemorrhage occurred in over $60 \%$ of patients after 1 replacement. The financial cost of occlusion averages over $\$ 600$ per patient. Therapeutic anticoagulation and smaller EVD catheters were statistically significant predictors of permanent occlusion, while nonideal catheter position was a marginally significant predictor. This study suggests that developing strategies or devices to prevent EVD occlusion, as well as preferential selection of larger diameter catheters, may be beneficial in reducing the burden associated with ventriculostomy malfunction.

\section{References}

1. Abla AA, Zabramski JM, Jahnke HK, Fusco D, Nakaji P: Comparison of two antibiotic-impregnated ventricular catheters: a prospective sequential series trial. Neurosurgery 68:437-442, 2011

2. Bauer DF, McGwin G Jr, Melton SM, George RL, Markert JM: The relationship between INR and development of hemorrhage with placement of ventriculostomy. J Trauma 70:1112-1117, 2011

3. Bogdahn U, Lau W, Hassel W, Gunreben G, Mertens HG, Brawanski A: Continuous-pressure controlled, external ventricular drainage for treatment of acute hydrocephalusevaluation of risk factors. Neurosurgery 31:898-904, 1992

4. Chi H, Chang KY, Chang HC, Chiu NC, Huang FY: Infections associated with indwelling ventriculostomy catheters in a teaching hospital. Int J Infect Dis 14:e216-e219, 2010

5. Fichtner J, Güresir E, Seifert V, Raabe A: Efficacy of silverbearing external ventricular drainage catheters: a retrospective analysis. J Neurosurg 112:840-846, 2010

6. Gardner PA, Engh J, Atteberry D, Moossy JJ: Hemorrhage rates after external ventricular drain placement. J Neurosurg 110:1021-1025, 2009

7. Huyette DRTB, Turnbow BJ, Kaufman C, Vaslow DF, Whiting BB, Oh MY: Accuracy of the freehand pass technique for ventriculostomy catheter placement: retrospective assessment using computed tomography scans. J Neurosurg 108:88-91, 2008

8. Kakarla UK, Kim LJ, Chang SW, Theodore N, Spetzler RF: Safety and accuracy of bedside external ventricular drain placement. Neurosurgery 63(1 Suppl 1):ONS162-ONS167, 2008

9. Khanna RK, Rosenblum ML, Rock JP, Malik GM: Prolonged external ventricular drainage with percutaneous long-tunnel ventriculostomies. J Neurosurg 83:791-794, 1995 
10. Ko JK, Cha SH, Choi BK, Lee JI, Yun EY, Choi CH: Hemorrhage rates associated with two methods of ventriculostomy: external ventricular drainage vs. ventriculoperitoneal shunt procedure. Neurol Med Chir (Tokyo) 54:545-551, 2014

11. Krötz M, Linsenmaier U, Kanz KG, Pfeifer KJ, Mutschler W, Reiser M: Evaluation of minimally invasive percutaneous CT-controlled ventriculostomy in patients with severe head trauma. Eur Radiol 14:227-233, 2004

12. Lo CH, Spelman D, Bailey M, Cooper DJ, Rosenfeld JV, Brecknell JE: External ventricular drain infections are independent of drain duration: an argument against elective revision. J Neurosurg 106:378-383, 2007

13. Mahan M, Spetzler RF, Nakaji P: Electromagnetic stereotactic navigation for external ventricular drain placement in the intensive care unit. J Clin Neurosci 20:1718-1722, 2013

14. Maniker AHVA, Vaynman AY, Karimi RJ, Sabit AO, Holland B: Hemorrhagic complications of external ventricular drainage. Neurosurgery 59 (4 Suppl 2):ONS419-ONS425, 2006

15. Olson DM, Zomorodi M, Britz GW, Zomorodi AR, Amato A, Graffagnino C: Continuous cerebral spinal fluid drainage associated with complications in patients admitted with subarachnoid hemorrhage. J Neurosurg 119:974-980, 2013

16. Patil V, Lacson R, Vosburgh KG, Wong JM, Prevedello L, Andriole K, et al: Factors associated with external ventricular drain placement accuracy: data from an electronic health record repository. Acta Neurochir (Wien) 155:1773-1779, 2013

17. Rahman M, Whiting JH, Fauerbach LL, Archibald L, Friedman WA: Reducing ventriculostomy-related infections to near zero: the eliminating ventriculostomy infection study. Jt Comm J Qual Patient Saf 38:459-464, 2012

18. Rosenbaum BP, Vadera S, Kelly ML, Kshettry VR, Weil RJ:
Ventriculostomy: Frequency, length of stay and in-hospital mortality in the United States of America, 1988-2010. J Clin Neurosci 21:623-632, 2014

19. Sussman ES, Kellner CP, Nelson E, McDowell MM, Bruce SS, Bruce RA, et al: Hemorrhagic complications of ventriculostomy: incidence and predictors in patients with intracerebral hemorrhage. J Neurosurg 120:931-936, 2014

20. Tse Ts, Cheng K, Wong K, Pang K, Wong C: Ventriculostomy and Infection: A 4-year-review in a local hospital. Surg Neurol Int 1:47, 2010

\section{Disclosure}

The authors report no conflict of interest concerning the materials or methods used in this study or the findings specified in this paper.

\section{Author Contributions}

Conception and design: Fargen. Acquisition of data: Fargen, O'Connor. Analysis and interpretation of data: Fargen, Neal, O'Connor, Murad. Drafting the article: Fargen. Critically revising the article: Fargen, Hoh, Rivera-Zengotita, Murad. Reviewed submitted version of manuscript: Fargen, Hoh, O'Connor, RiveraZengotita, Murad. Approved the final version of the manuscript on behalf of all authors: Fargen. Statistical analysis: Neal. Study supervision: Fargen, Murad.

\section{Correspondence}

Kyle M. Fargen, Department of Neurosurgery, Medical University of South Carolina, 96 Jonathan Lucas St., CSB 301, Charleston, SC 29425. email: fargen@musc.edu. 